\title{
Les activités artistiques dans le projet de soin en santé mentale
}

\author{
Julie VANDEWALLE ${ }^{1}$ et ISABelle CABY $^{2}$ \\ ${ }^{1}$ Direction Départementale de la Cohésion Sociale du Pas-de-Calais, France \\ ${ }^{2}$ Univ. Artois, Univ. Lille, Univ. Littoral Côte d'Opale, EA 7369, Unité de Recherche Pluridisciplinaire \\ Sport Santé Société (URePSSS), F-62800 Liévin, France \\ Fédération Française de Sport Adapté
}

\section{Écho de la communauté • Echoes of the Community}

\section{Résumé}

Que faire quand le corps n'existe plus, qu'il a disparu, qu'il a perdu sa place, son unité? La différence, la souffrance psychique sont stigmatisantes. L'exclusion est là, pesante, présente et l'isolement, une conséquence. Dans ce cas, la mise en scène du corps peut représenter un outil thérapeutique efficace pour la prise en charge et l'accompagnement de sujets en situation de souffrance psychique atteints par exemple de dépression, de psychose, de dépendance, de mélancolie, de bipolarité, d'état limite ou encore de schizophrénie. Si la danse permet d'accéder à un univers artistique où les émotions sont centrales, le défilé chorégraphique nous a permis d'apporter, aux sujets en détresse, confiance en soi et conscience de soi. Le corps devient alors un véritable support à la médiation, à la (re)construction de liens physiques, psychiques et sociaux. En ce sens, les activités artistiques, la création artistique et le processus de résilience associé s'inscrivent dans une approche globale de santé mentale.

Mots-clés : handicap, santé mentale, sport adapté, activités artistiques, projet de soin

\begin{abstract}
What to do when the body no longer exists, he disappeared, he lost his place, his unit. The difference, the psychic suffering are stigmatizing. Exclusion is heavy, and the isolation is a systematic issue. In this case, body staging can be an effective therapeutic tool to support and accompaniment of psychic suffering subjects such as depression, psychosis, addiction, melancholy, bipolar, limit State or schizophrenia. If dance allows access to an artistic World where emotions are central, the choreographic parade allowed us to bring to the topics in mental distress, self-confidence and selfawareness. he body then becomes a true support to mediation, the (re) construction of physical, psychic and social links. Thus, artistic activities, artistic creation, the associated resilience process fit into a comprehensive approach to mental health.
\end{abstract}

Keywords : disability, mental health, adapted sport, artistic activities, care project 


\section{Introduction}

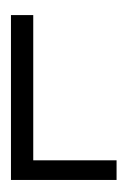

e projet de défilé de mode chorégraphique «Je m'défile » est une ouverture de personnes en souffrance psychique vers des activités extérieures aux soins. Ce projet artistique est une réponse au besoin de ces personnes de se réapproprier une image erronée qu'elles ont d'elles-mêmes.

« Je m'défile » constitue un vrai dualisme opposant la particularité des activités artistiques et la spécificité du handicap psychique. C'est le passage d'un corps caché, meurtri, oublié, douloureux à un corps affiché, vivant, pensé et joyeux. Une réponse à l'isolement, à la difficulté éprouvée dans la relation à l'autre, à soi; l'expression d'un corps nouveau, tendant à se reconstruire et se projeter dans l'avenir.

« Je m'défile », c'est eux, c'est nous, et c'est une invitation à entrer dans la danse ».

\section{Le choix des activités artistiques en santé mentale}

La difficulté de définir les activités artistiques et le côté abstrait de leurs fondements peuvent interroger sur les bénéfices et les risques d'utiliser ce type de support dans le milieu de la santé mentale.

Si l'on reprend le concept de création, celui-ci peut s'avérer être un point fort quant à la possibilité, pour le pratiquant, d'exprimer ses ressentis avec une nouvelle forme de communication. Cette matérialisation de la pensée offre l'occasion de transmettre un message personnel à autrui. Elle permet également à chacun d'interpréter le message offert par l'autre et ouvre alors le champ de l'imaginaire. II y a donc, en ce sens, une relation indirecte à autrui qui se crée, permettant ainsi de lutter contre l'isolement dont les usagers sont souvent victimes. Néanmoins, chez des sujets psychotiques dont les limites corporelles sont floues, n'y a-t-il pas un danger d'ancrer la personne dans une mauvaise représentation de son corps, dans une vision déformée de celui-ci? Le message reçu peut également être différent de celui émis puisque la force du message sera libre d'interprétation par celui qui le reçoit. Se peut-il que ce message soit mal interprété?

Tout comme pour la création, l'imagination engagée par l'acte créatif montre une ambiguïté sur l'utilité des activités artistiques chez le sujet suivi pour un trouble psychique. D'un côté, elle stimule la pensée, en incitant la personne à chercher dans un "réservoir de pensées » d'images connues. Cela permet au sujet d'engager une réflexion propre sur son ressenti et d'en débattre avec le groupe. D'où l'importance de laisser un temps aux pratiquants, après une situation engageante ou une séance, pour s'exprimer verbalement sur ce qu'ils ont vécu. Outre l'échange avec le groupe, cela permet également au pratiquant de prendre du recul sur les choses en les mettant à distance. D'un autre côté, l'imagination se définit aussi comme la faculté de changer les images fournies par la perception (Wunemburger, 1991). Le «foyer d'illusions " qu'elle constitue peut représenter un danger consistant à entretenir les distorsions de la pensée, caractéristiques chez certains sujets atteints de troubles psychiques.

Enfin, le questionnement du corps par l'acte symbolique permet une autre forme d'expression où le sujet se réapproprie son corps. La signification première des choses, traduite par les codes sociaux plus ou moins connus de tous, laisse la place au sens que l'on veut donner à ce qui est représenté. Même si le danger de transformation du réel existe, la relation à l'autre qui s'installe, et notamment au soignant, a son importance pour veiller à ne pas entretenir l'irréel.

Le fait d'intégrer le soignant à l'activité lui permet ainsi de créer un autre lien avec l'usager et d'éviter d'installer la personne dans de fausses représentations où se confondent réalité et imaginaire. Cette position thérapeutique est basée sur une réciprocité de la confiance, et ce afin que l'autre apprenne de lui et pour lui. Ainsi la barrière soignant/soigné s'efface tout comme le rapport de force qui peut parfois s'installer. II s'agit d'amener chacun à s'étonner et à se laisser étonner par l'autre. 
Dans ce contexte, il semble que la complémentarité entre le soignant et l'intervenant soit indispensable afin d'extraire les richesses de l'activité sans aller à l'encontre du processus soignant, mais au contraire en le renforçant. En disposant des tapis dans la salle de manière à ce qu'il y en ait un par personne et sans chercher à dissocier les soignants des soignés, l'intervenant propose un cadre propice à la création d'une unité de groupe. Ainsi, chacun reçoit une proposition de démarrer l'activité dans un espace personnel parmi les autres. C'est d'ailleurs un autre intérêt d'utiliser ce type d'activités par rapport à un sport plutôt collectif qui imposerait une relation à l'autre ou un sport individuel qui pourrait renforcer le côté isolant de la pathologie psychique. Les activités artistiques permettent de proposer une activité pour soi, mais avec les autres. L'intervenant prend également place et s'inclue dans le groupe qu'il essaye de constituer, sans pour autant omettre qu'il doit garder un rôle d'observateur extérieur afin de ne pas perdre la prise de recul nécessaire pour analyser et réguler son intervention. Dans cette idée, l'activité physique ne soigne pas, mais propose une prise en charge globale qui peut faire partie d'un processus de soin, alors même qu'elle est souvent perçue comme une simple activité de loisirs.

\section{De l'analyse du public au choix de l'activité}

II existe de très nombreux tableaux cliniques accompagnant une grande diversité de psychopathologies. Même s'il est important d'analyser le public pour choisir de manière cohérente et appropriée une activité physique adaptée, il parait tout à fait incohérent de vouloir classer les personnes dans une description clinique trop stéréotypée.

II existe néanmoins quelques traits caractéristiques à prendre en compte chez ces personnes (Rangaswamy Srinivasa Murphy and al. Rapport sur la santé dans le monde, 2001), comme des traits dépressifs plus ou moins marqués avec une perte d'intérêt pour les activités ou un désintérêt général de la personne. On retrouve aussi souvent des troubles de l'estime de soi et de la confiance en soi profondément altérées ou non stabilisées comme dans le cas d'une bipolarité. Enfin, et c'est là un intérêt majeur des activités physiques adaptées, la détresse psychique caractéristique chez ces personnes tend à les isoler de leur famille et de la société. Or, les activités artistiques ont pour finalité d'être vues et offertes à un public. L'idée de créer un projet chorégraphique s'est alors posée comme une réponse à l'isolement du sujet pouvant amener ce dernier à reconsidérer sa place dans son environnement. Une place perdue d'une part à cause de la pathologie psychique, mais aussi de par l'exclusion d'une société qui, par méconnaissance, rejette l'individu ne répondant pas à un système symbolique commun.

En effet, la réalité commune donne une signification aux objets, aux espaces, au temps, aux actes, aux autres et à nous-mêmes. C'est ce système symbolique qui permet aux personnes de se comprendre entre elles. La perte de contact avec cette réalité, commune chez le sujet psychotique, rend donc difficile la relation à l'autre. Elle s'explique par la perte d'unité corporelle rendant les limites du corps floues (Couvez, 2009). Les parties du corps ne sont plus reliées entre elles pour former un tout. Le corps devient alors étranger, morcelé et « déshabité ". C'est ce qui rend le travail avec le miroir (très utilisé en danse) difficile, voire impossible. II faut donc veiller à adapter l'activité aux particularités du public, par exemple lors d'un exercice de relaxation utilisant la technique de contraction-relâché, où il est d'autant plus indispensable de terminer par une contraction du corps dans son ensemble, au risque d'aggraver le phénomène de morcellement.

Cette notion de corps étranger explique la perte de sens général de l'espace puisque c'est à partir de cet espace corporel que s'organise psychiquement l'espace environnant. En perdant cet espace environnant, le sujet psychotique perd également la notion de temps qui est une résultante du mouvement dans l'espace.

L'unité corporelle se dissocie de l'unité psychique entraînant une perte d'individualité. La vie désaffectée de l'individu psychotique rend alors son monde sans aucun sens et tend au repli

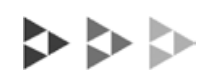


sur soi. Cette perte d'individualité, ajoutée à l'exclusion sociétale que peuvent subir les personnes en souffrance psychique, entraîne des troubles de l'estime de soi car aucun regard n'est neutre. Et c'est le mélange des regards et des jugements que l'on porte sur soi qui constitue l'estime de soi.

D'après Christophe Andre (2009), parmi les nombreux symptômes de souffrance de l'estime de soi, on retrouve notamment: l'obsession de soi, le sentiment de solitude, la difficulté à demander de l'aide et le caractère excessif des émotions négatives. Autant de symptômes qui sont un facteur de risque pouvant amener à la dépression, que l'on retrouve fréquemment chez des personnes atteintes de troubles psychiques.

On comprend alors la spirale dans laquelle peut tomber une personne atteinte de troubles psychiques : entre isolement, exclusion et troubles de l'estime de soi qui se renforcent l'un et l'autre.

Les activités artistiques sont ici un moyen de travailler les sensations corporelles et la composante espace-temps. En les situant dans une démarche visant à sortir la personne de cette spirale d'exclusion et d'isolement, on fait le choix d'emprunter à une activité ses fondements pour répondre aux besoins identifiés dans l'analyse du groupe. C'est donc sur la base de ces traits communs que s'est porté le choix de l'activité. II faut également veiller à respecter les caractéristiques biologiques et démographiques (moyenne d'âge, sexe, vécu sportif, etc.) pour proposer une démarche cohérente.

\section{De l'analyse du public à « Je m'défile »}

En prenant en compte les facteurs biologiques, les souhaits des personnes ayant participé au projet « Je m'défile » d'avoir une activité visant à s'entretenir, sans oublier les caractéristiques communes à leurs syndromes (dépression, psychose, dépendance, mélancolie, bipolarité, état limite, schizophrénie, etc.), notre projet artistique visait de changer le regard qu'a la personne sur elle-même, de lui redonner une con- fiance en soi perdue, et ce, grâce à un investissement de la personne pour elle-même; cet investissement se faisant sur une période définie au long terme et ayant pour finalité de tendre à l'acceptation de soi.

Ce processus de choix de l'activité donne alors un sens à la pratique qui est indispensable dans un cadre de soin. Pour ce faire, nous avons imaginé plusieurs étapes dans la construction de ce projet artistique. Une première phase de redécouvertes, voire de découvertes pour certains: la prise de conscience corporelle, la recherche de nouvelles sensations, la place du corps dans l'espace, le rapport à son propre corps pour arriver au rapport à l'autre. Une fois ces bases posées, une seconde étape visait à approfondir les acquis : l'acceptation de soi, la connaissance de ses forces et faiblesses pour ensuite se tourner vers le travail avec autrui. Enfin, une dernière phase de travail chorégraphique avait pour but d'amener : l'acceptation d'être une individualité dans un groupe, l'utilisation de son corps comme moyen d'expression personnelle et enfin l'expression commune d'un message transmis par le groupe au public.

De cette analyse ont été choisies certaines activités artistiques ou gymniques nous permettant d'aboutir à la préparation du défilé de mode chorégraphique : le stretching, la danse, l'expression corporelle, la gymnastique rythmique ou encore le renforcement musculaire.

L'activité d'expression corporelle et la danse ont été les plus utilisées en raison de l'éventail des possibilités qu'elles offrent (Figure 1). Des différentes situations que nous avons pu mettre en place, certaines sont devenues des créations à part entière présentées lors du défilé de mode chorégraphique.

C'est le cas d'une situation qui vise à constituer l'écoute du groupe. II s'agit pour le groupe de former une file indienne qui déambule dans l'espace où le premier danseur choisit le déplacement en fonction de la musique et de son ressenti. II décide de s'arrêter et le groupe doit se réunir pour former une grappe pour laquelle la seule consigne est d'avoir au minimum un 
contact avec un membre du groupe. Une fois la grappe installée, un membre du groupe choisit de repartir en définissant son déplacement et sans l'annoncer au préalable. La file indienne se reconstitue alors et, de nouveau, l'arrêt sera marqué pour former une autre grappe. On constate d'ailleurs en faisant cette situation que les premières grappes sont très linéaires et les contacts se font uniquement par la main, avec une certaine distance entre chaque danseur. Plus la situation est répétée, plus on constate une modification de la grappe qui se resserre, qui a des différences de niveaux (sol, debout, à genoux, etc.) et dont les contacts sont variés. De cette situation a découlé un travail sur les expressions où chaque grappe représentait un tableau expressif autour de la colère, de la joie, de la tristesse, etc. Mise en musique, cette situation est devenue une chorégraphie à part entière. Ainsi, le passage des activités à l'élaboration du défilé s'est fait naturellement et dans la continuité du projet.

Un autre versant du projet s'attardait sur les costumes du défilé. L'image du corps et le sentiment d'appartenance à un groupe sont corrélés à l'apparence physique et à la tenue vestimentaire. II nous a paru important de ne pas négliger cette partie-là du défilé et, au-delà des partenariats locaux avec des créateurs ou stylistes nous ayant mis à disposition leurs créations, nous avons choisi de travailler avec une artiste textile sur des ateliers de tissage. Ainsi, les usagers ont préparé des moules en plâtre recouverts de tissus tissés par leurs soins. Ces créations de «faux culs" (rembourrages que les femmes portaient sous leur robe pour augmenter le volume apparent de leur postérieur) et bustiers ont ajouté une valorisation supplémentaire à leur implication dans le projet. On a pu ressentir une certaine fierté de porter ces créations lors du défilé, autour d'une mise en scène réalisée de manière à les mettre en valeur. Au-delà de cette fierté, on a constaté chez certaines personnes qu'elles venaient de manière plus apprêtée aux séances. Se maquiller, se coiffer, s'habiller... autant de choses du quotidien qui n'existaient plus et qui, par le biais du projet, ont repris leur place. On comprend alors que la portée du projet ne s'arrête pas à la séance hebdomadaire d'activité et au défilé chorégraphique : les impacts se ressentent sur le quotidien de l'ensemble du groupe.

\section{Les limites de ce choix d'activités au sein du projet}

Peter Brook (2016) définit l'art comme « (...) un effort de l'Homme pour se révéler aux autres. II permet au niveau individuel de poser sa propre référence, d'affirmer sa distinction, de prendre la parole et de la défendre. Mais comme il n'y a pas d'écriture sans lecture, il n'y a d'Art que par et pour autrui ». Le projet défilé de mode chorégraphique, comme toute forme d'art, a pour finalité d'être vu. Le fait de se mettre en représentation a été un aspect délicat à instaurer au sein du groupe puis à anticiper et à accompagner jusqu'à l'après-défilé. La particularité des activités physiques artistiques est l'engagement émotionnel du pratiquant. Se montrer, défiler, danser peut donc sembler contradictoire avec les troubles de l'image du corps et les difficultés que peuvent éprouver les pratiquants. Sauf si on considère ces activités comme un outil pour se dépasser, prendre sur soi, avancer.

Cette idée de défiler ne s'est pas glissée dès le début des séances, mais quelques semaines plus tard, au moment où une bonne cohésion de groupe s'installait, permettant ainsi aux plus optimistes d'entraîner naturellement les plus réticents à s'investir dans le projet. Une fois l'ensemble du groupe porté par l'émulation collective, il a fallu gérer une phase de stress de plus en plus importante à mesure que le défilé approchait. Pour dédramatiser cette représentation finale, il semble important d'instaurer un principe de bienveillance, et ce, dès les premières séances, sur l'ensemble du cycle. Au-delà de ce principe, le positionnement de l'intervenant vis-à-vis du groupe est là aussi primordial. Tout comme pour l'installation des tapis disposés dans tout l'espace sans distinction pour l'ensemble des participants (soignés, soignants ou intervenant), l'intervenant doit s'engager pleinement dans des situations pour banaliser le regard d'autrui aux yeux du groupe. Les participants s'engagent plus dans l'activité si tout le monde participe et accepte d'être vu.

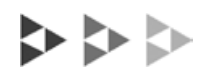




\section{FIGURE 1}

\begin{tabular}{|c|c|c|}
\hline Activité & Définition & Apports/Bénéfices \\
\hline Relaxation & $\begin{array}{l}\text { Elle se pratique selon différentes techniques (train- } \\
\text { ing autogène de Schultz, Méthode Vittoz, etc.). } \\
\text { Lors du cycle d'activités, elle sera plutôt abordée } \\
\text { épisodiquement sous différents principes : contrac- } \\
\text { tion-relâchement, sensations corporelles tactiles, } \\
\text { chaleur-lourdeur, respiration abdominale- } \\
\text { thoracique. }\end{array}$ & $\begin{array}{l}\text { La relaxation prépare le corps à la séance } \\
\text { et permet la redécouverte de celui-ci à } \\
\text { travers la recherche de nouvelles sensa- } \\
\text { tions. Elle permet d'utiliser le vécu subjectif } \\
\text { des sensations corporelles comme base de } \\
\text { support à l'élaboration d'une nouvelle } \\
\text { image de soi plus positive. }\end{array}$ \\
\hline Stretching & $\begin{array}{l}\text {... ou étirement. } \\
\text { II a pour but d'assouplir ou de gagner en amplitude } \\
\text { articulaire. C'est un moyen de préparer le corps à } \\
\text { l'exercice et de favoriser sa récupération. }\end{array}$ & $\begin{array}{l}\text { Le stretching va, d'une part, préparer le } \\
\text { corps à la séance et, d'autre part, permettre } \\
\text { un allongement et une prise de conscience } \\
\text { de l'allongement corporel. } \\
\text { De plus, la sensation d'étirement contribue } \\
\text { au bien-être des personnes qui ne sentent } \\
\text { plus les tensions. }\end{array}$ \\
\hline Danse & $\begin{array}{l}\text { C'est un art corporel où le corps peut se mouvoir, } \\
\text { en accord avec le temps et l'espace. Elle peut se } \\
\text { pratiquer selon différentes techniques, mais elle } \\
\text { reste caractérisée par une suite de mouvements } \\
\text { plus ou moins prédéfinis. } \\
\text { Différents supports musicaux seront utilisés lors de } \\
\text { ce cycle, afin que chacun puisse trouver un style } \\
\text { qui lui convient et sur lequel il pourra travailler. }\end{array}$ & $\begin{array}{l}\text { La danse permet de situer son corps dans } \\
\text { l'espace, d'une manière individuelle et/ou } \\
\text { collective. } \\
\text { Elle construit une autre organisation tempo- } \\
\text { relle, en lien avec un support musical. } \\
\text { Enfin, l'utilisation du corps dans ses diffé- } \\
\text { rentes formes et énergies apporte une } \\
\text { maîtrise de celui-ci plus importante. } \\
\text { Le contrôle du mouvement et } \\
\text { l'interprétation qu'on lui donne permettent } \\
\text { un nouveau mode d'expression. } \\
\text { Toutes ces nouvelles possibilités tendent à } \\
\text { aimer ce « nouveau » corps. }\end{array}$ \\
\hline $\begin{array}{l}\text { Expression } \\
\text { corporelle }\end{array}$ & $\begin{array}{l}\text { C'est l'extériorisation d'une pensée, d'une sensa- } \\
\text { tion, d'un sentiment au travers du corps. Cette } \\
\text { expression peut se faire par un geste, un mouve- } \\
\text { ment ou simplement par une mimique. } \\
\text { Ici, elle a pour but de libérer la personne de ses } \\
\text { pensées intrusives ou de ses émotions négatives. } \\
\text { Elle sera utilisée à travers la danse, la gymnas- } \\
\text { tique rythmique ou encore le mime. }\end{array}$ & $\begin{array}{l}\text { Elle permet de communiquer autrement } \\
\text { que par les mots et d'évacuer les tensions } \\
\text { que l'on peut ressentir. La personne peut } \\
\text { alors apprendre à gérer son émotion, à la } \\
\text { percevoir, l'accepter, et la contrôler. } \\
\text { Cette gestion des émotions contribue au } \\
\text { bien-être de la personne. }\end{array}$ \\
\hline Mime & $\begin{array}{l}\text { Le mime est une forme de théâtre composée } \\
\text { d'attitudes, de gestes et de mimiques. Le langage } \\
\text { du corps est utilisé pour exprimer un morceau de } \\
\text { vie. } \\
\text { Le mime, à travers ce cycle, est utilisé comme } \\
\text { moyen d'expression corporelle où même un corps } \\
\text { statique est un corps vivant. }\end{array}$ & $\begin{array}{l}\text { Cette concentration sur soi permet un rap- } \\
\text { port au corps différent, une maîtrise cons- } \\
\text { tante qui ne laisse pas la place au hasard. } \\
\text { L'apprentissage de cette présence scé- } \\
\text { nique permanente peut tendre à gagner en } \\
\text { assurance et ainsi renforcer une estime de } \\
\text { soi affaiblie. }\end{array}$ \\
\hline $\begin{array}{l}\text { Gymnastique } \\
\text { rythmique }\end{array}$ & $\begin{array}{l}\text { Cette discipline artistique, à travers l'utilisation de } \\
\text { cinq engins, se caractérise par une composition } \\
\text { chorégraphique réalisée sur un support musical. } \\
\text { Pour ce cycle, la GR aborde essentiellement } \\
\text { l'aspect relationnel grâce aux engins. }\end{array}$ & $\begin{array}{l}\text { La GR permet d'aborder la relation à autrui } \\
\text { à travers les engins. L'aspect collectif pour- } \\
\text { ra ensuite être développé et permettre } \\
\text { d'apprendre à travailler ensemble en créant } \\
\text { une unité au sein du groupe. } \\
\text { Cet aspect relationnel est très important, du } \\
\text { fait de l'isolement dont sont touchées les } \\
\text { personnes et prend donc dans ce cycle tout } \\
\text { son sens. }\end{array}$ \\
\hline
\end{tabular}


Malheureusement, chez certaines personnes comme celles suivies pour une dépendance, la semaine précédant le défilé est anxiogène et peut entraîner une prise de produits pour compenser cette angoisse. II a fallu gérer des conduites déviantes la veille du défilé chorégraphique, ce qui a été possible grâce au dialogue instauré par les infirmières avec les usagers et grâce à la relation entre l'intervenant et le soignant. L'importance du dialogue et de la complémentarité entre l'équipe soignante et l'intervenant est primordiale pour gérer ce genre de situation.

Une autre limite, perçue à la suite du défilé, concerne cette relation soignant-soigné-intervenant. Plus le défilé approche, plus l'implication du groupe augmente à travers des répétitions nombreuses et un investissement émotionnel important. La relation du groupe peut prendre une tournure différente au niveau des liens qui en unissent les membres. Lors du bilan, certains participants ont évoqué le fait d'avoir été jusqu'au bout du projet «pour faire plaisir " à l'intervenant ou au groupe et non pour eux-mêmes. En utilisant des activités mettant en jeu des émotions et demandant aux personnes de s'exprimer, il existe en effet un risque d'engager trop loin la relation, et que celle-ci, tôt ou tard, soit néfaste pour les usagers. C'est ainsi qu'à l'arrêt du projet, certains ont eu une phase difficile à gérer.

L'après-défilé est d'ailleurs une étape clé à prendre en compte dès le début du projet. En effet, tout comme il est important d'accompagner les personnes dans la gestion du stress lié à l'approche de l'évènement, il est indispensable de surveiller et d'anticiper la fin du projet, où le sujet mis en pleine lumière, redevient un individu parmi d'autres. Et pour certaines personnes, comme celles dont l'exacerbation des traits de caractère était amplifiée au même titre que la prise de produits lors d'une représentation scénique, la phase qui suit le défilé peut être source de décompensation. Le bénéfice de l'activité et du projet dans sa globalité est alors considérablement réduit et peut impacter l'ensemble du groupe.
À contrario, « Je m'défile » était, pour d'autres, un tremplin vers de nouveaux projets. Une personne ayant subi une dépression réactionnelle à la suite d'un événement traumatisant nous a expliqué avoir repris goût à son quotidien. Elle souhaitait de nouveau sortir de chez elle dans le but de s'investir auprès d'une association, au sein de laquelle elle se sent utile. Quelques mois après le défilé, cette personne ne sentait plus le besoin de venir au centre médico-psychologique.

On comprend alors que la frontière entre bénéfice et limite reste fragile concernant l'utilisation des activités physiques et artistiques avec un public en situation de handicap psychique. Néanmoins, comme souvent dans le milieu des activités physiques et sportives, il s'agit de trouver le bon équilibre entre les besoins de la personne, le sens que l'on souhaite donner à l'activité et les apports de cette dernière en fonction des démarches pédagogique et didactique employées. II semble important de rester à l'écoute des usagers, mais aussi des personnes qui les entourent : famille, médecin, kinésithérapeute, etc.

Dans le cadre du projet, les activités artistiques nous semblaient être un bon moyen pour amener les personnes à reconsidérer leur place dans leur environnement et améliorer leur quotidien. Très vite, la danse et l'expression corporelle ont pris une place prépondérante dans les séances, de par l'impact qu'elles ont sur l'image du corps au travers de la création.

\section{La danse et la création artistique comme projet de soin}

La danse, définie comme un art de se mouvoir (Commande, 2011), est donc une activité de symbolisation motrice, amenant un message transmis par le corps qui devient support d'expression. Dans ce processus, le sujet est amené à chercher de nouvelles sensations, à (re)découvrir le corps pour pouvoir ensuite construire ce nouveau langage corporel.

En reprenant la logique d'activité de cette discipline, à savoir l'élaboration d'un langage corporel symbolique, personnel et abstrait, porteur

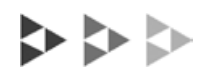


d'un message offert à l'interprétation du public, on considère le sujet au-delà de sa simple présence motrice en le plaçant dans un rapport au monde où sa présence est sensitive, affective et relationnelle.

De ce fait, cette prise de conscience du mouvement dansé comme forme d'expression et non comme exécution technique amène le sujet à considérer son corps autrement. La danse, d'un point de vue créatif et non simplement exécutif, privilégie donc un rapport au corps qui va au-delà de l'utilitaire. Le geste dansé dépasse l'exercice physique et offre une conscience du « faire » et du « moi faisant ». Dans cette approche, le danseur va utiliser son corps pas simplement comme outil, mais comme représentant de sa pensée. De plus, certaines techniques de médiation corporelle, comme la relaxation ou le stretching, amènent une écoute du corps. L'engagement du vécu subjectif des sensations corporelles va offrir une base de support à l'élaboration d'une nouvelle image de soi et permettre ainsi une redécouverte de son corps.

En utilisant ses gestes pour donner du sens à ses émotions, le sujet peut alors recréer un lien perdu avec autrui. C'est donc le corps qui sert d'objet médiateur dans la relation à l'autre. Cette médiation corporelle se veut un intermédiaire entre soi et l'autre. L'espace proposé par la médiation permet de partager avec autrui quelque chose de commun qui devient alors témoin de la relation existante entre deux personnes.

Tout comme les activités artistiques peuvent être vues comme un support de soin agissant sur l'enveloppe corporelle du sujet, la création artistique peut apporter des éléments de réponse à la problématique de la perte des notions d'espace et de temps chez le sujet psychotique.

Selon Paul Valery, la danse est une action de l'ensemble du corps humain transposée dans un espace/temps qui n'est plus tout à fait le même que celui de la vie pratique. Le corps concrétise le mouvement par le biais de trois composantes : le temps, l'espace et l'énergie.
L'approche du tableau clinique de la psychose sous l'angle de ces trois composantes amène à considérer les activités artistiques comme un support pouvant favoriser une unité psychique, corporelle et spatio-temporelle. La création artistique amène alors une construction à la fois personnelle et collective d'un espace défini, d'un univers symbolique commun aux interprètes, d'un monde sonore et donc temporel, et d'un mode de communication spécifique.

\section{La création artistique comme processus de résilience?}

Ce processus de création est utilisé comme médiateur entre l'individu en souffrance et la société devant laquelle il s'exprime autrement. Ces outils lui permettent de créer un nouveau langage où l'expression permet la maîtrise de l'émotion et la mise à distance du traumatisme. La capacité de résilience de ces personnes pourrait-elle être renforcée par cette nouvelle forme d'expression? Parmi les trois grandes familles de facteurs de protection contribuant à la résilience, à savoir les facteurs individuels, les facteurs familiaux et les facteurs de soutien, les activités artistiques peuvent avoir une place importante dans le renforcement de certains d'entre eux, notamment au niveau individuel.

En plus de révéler les compétences créatives de l'individu qui va développer une nouvelle forme d'expression de son vécu et de ses émotions, la planification sur le long terme d'un défilé chorégraphique ou de toute autre forme de production artistique permet d'engendrer une projection dans l'avenir et un sentiment de signification. Le sentiment d'existence ou celui d'avoir une place dans la société pourra avoir un impact sur l'optimisme du sujet en situation de handicap psychique, sur sa croyance en une issue positive.

\section{Conclusion}

Que faire quand le corps n'existe plus, qu'il a disparu, perdu sa place, son unité? Comment dépasser la stigmatisation de cette souffrance psychique considérée comme une différence qu'il faut exclure? 
Le pari de « Je m'défile » est celui de mettre en scène des corps meurtris, en détresse, pour les amener vers une (re)construction de liens physiques, psychiques et sociaux. II n'est pas une fin en soi, mais se veut un moyen d'accompagner un individu dans son projet de soin, en s'inscrivant dans une approche globale de santé mentale. Cette mise en scène du corps, véritable support à la médiation, laisse la place aux émotions et libère le geste qui remplace la parole. Si le défilé chorégraphique a permis d'apporter aux sujets confiance en soi et conscience de soi, on admet qu'il puisse être une issue plus ou moins positive pour certains, une réponse pour d'autres, une étape pour tous.

\section{Références}

ANDRÉ, C. (2006). Imparfaits, libres et heureux : pratiques de l'estime de soi. France : Éditions Odile Jacob.

Assoun, P. L. (2003). La résilience à l'épreuve de la psychanalyse. Synapse, 198, 25-28.

BARANES, C. P. (2007). Des thérapies à médiation corporelle pour les adolescents. Un exemple : la relaxation. Neuropsychiatrie de l'enfance et de l'adolescence, 55(2), 120-126.

Brook, P. (2016). L'espace vide. Écrits sur le théâtre. France : Éditions du Seuil.

Mondiale de LA SANTÉ, O. (2007). Rapport sur la santé dans le monde. Les risques sanitaires des adultes: les progrès marquent le pas et les disparités s'accroissent.

Viaud, O., \& Le Jalle, F. (2012) Le Compagnon de Jeu. (Trans'formation, Strasbourg)

WunEnburger, J. J. (1991). L'imagination (No. 649).

Presses Universitaires de France-PUF. 